\title{
A prospective study of metformin versus myoinositol plus d-chiroinositol combination therapy in polycystic ovarian syndrome
}

\author{
Sukhmani Jaura ${ }^{1}$, Inderpal Kaur ${ }^{1 *}$, Jatinder Singh ${ }^{1}$, Anita Madan ${ }^{2}$
}

\begin{abstract}
${ }^{1}$ Department of Pharmacology, ${ }^{2}$ Department of Obstetrics and Gynaecology, Government Medical College Amritsar, Punjab, India
\end{abstract}

\author{
Received: 03 December 2019 \\ Revised: 11 January 2020 \\ Accepted: 13 January 2020 \\ *Correspondence: \\ Dr. Inderpal Kaur, \\ Email: inderpalpharma@gmail.com
}

Copyright: (C) the author(s), publisher and licensee Medip Academy. This is an open-access article distributed under the terms of the Creative Commons Attribution Non-Commercial License, which permits unrestricted non-commercial use, distribution, and reproduction in any medium, provided the original work is properly cited.

\begin{abstract}
Background: Polycystic ovarian syndrome (PCOS) is one of the most common endocrine disorders in women of reproductive age. Insulin resistance and resultant hyperinsulinemia contribute to hyperandrogenism in these patients. Weight loss or pharmacologic interventions that lower insulin levels reduce androgen levels. This study was planned to evaluate efficacy of metformin versus myoinositol plus d-chiroinositol combination therapy in PCOS patients and its effects on clinical, hormonal and radio diagnostic dimensions.

Methods: This was a prospective study for nine months. 50 newly diagnosed PCOS patients from the Department of Obstetrics and Gynaecology, Government Medical College (GMC), Amritsar, were randomly divided into 2 groups. One group was given metformin $500 \mathrm{mg}$ twice daily and another myoinositol plus d-chiroinositol $1000 \mathrm{mg}$ twice daily for 9 months. Follow up was done at 3, 6, 9 months. At each visit, ultrasonography and hormone levels were evaluated. Informed consent was taken. The approval of the Institutional Ethics Committee, GMC, Amritsar was also obtained.

Results: The percentage change in free testosterone levels $(22.46 \pm 6.47)$ and insulin levels $(34.24 \pm 15.02)$ show statistically significant decrease in group $2(\mathrm{p}<0.001)$. There was statistically significant $(\mathrm{p}<0.05)$ fall in AMH levels $(22.41 \pm 7.78)$ in group 1 . There was no statistically significant difference between the 2 groups in ovarian volume on ultrasonography, random blood glucose levels, luteinizing hormone (LH), follicle-stimulating hormone (FSH) levels, estrogen and progesterone levels.

Conclusions: It was observed that both treatments are equally effective in the treatment of polycystic ovarian syndrome with better tolerability in myoinositol and d-chiroinositol group.
\end{abstract}

Keywords: PCOS, Metformin, Myoinositol, d-chiroinositol

\section{INTRODUCTION}

Hormonal disorder common among women of reproductive age is polycystic ovarian syndrome (PCOS) and globally its incidence ranges from 2.2-26\%. ${ }^{1}$ Hyperandrogenism in these patients is the result of insulin resistance and resultant hyperinsulinemia. Life style modification and pharmacological agents that lower insulin levels improve menstrual cycle regularity by further reducing androgen levels.
Metformin by decreasing insulin resistance improves ovulation in women with PCOS. $^{2}$ Myo-inositol a naturally occurring member of the vitamin B group regulates glucose transport and follicle-stimulating hormone signaling in the ovary whereas glycogen and insulin-induced androgen synthesis is mainly controlled through d-chiroinositol. ${ }^{3,4}$ Considering the alleviation of insulin excess by both the drugs metformin and myoinositol, the present study has been designed to study 
and compare the effects of these two drugs in patients with PCOS.

\section{METHODS}

The present study was prospective randomized parallel design interventional study. The study comprised of 50 newly diagnosed PCOS patients in the age group of 15 45 years in the outpatient department of Department of Obstetrics and Gynaecology at Bebe Nanki Centre for Mother and Child Care, Government Medical College (GMC), Amritsar. This study was conducted from April 2018 to September 2019. An informed consent was taken from all the patients after explaining study drugs, its benefits and side effects. The approval of the Institutional Ethics Committee, GMC, Amritsar was also obtained.

The diagnosis of the syndrome was made according to the Rotterdam's criteria in which at least two out of three criteria (hyperandrogenism, ovulatory dysfunction, and polycystic ovarian morphology) are needed to diagnose the disorder. ${ }^{5}$ Patients with history of hypertension and diabetes mellitus, women who received previous treatment with other drugs within last 6 months and pregnant females were excluded from the study.

\section{Study design}

All the newly diagnosed PCOS patients from Department of Obstetrics and Gynaecology at Bebe Nanki Centre for Mother and Child Care, Amritsar were recruited for the study. Baseline Tests were done at the start of therapy in all these patients. 50 patients meeting the inclusion criteria were selected. Systemic disease, other comorbidities excluded.

Subjects selected were randomly divided by Simple Randomization method into 2 groups such as Group 1 with 25 patients on Metformin $500 \mathrm{mg}$ BD daily and Group 2 with 25 patients on Myoinositol and dchiroinositol $1000 \mathrm{mg}$ BD daily. Follow up done at $3^{\text {rd }}$, $6^{\text {th }}, 9^{\text {th }}$ month to evaluate the results of the drugs on the hormone levels of the subjects and if any adverse effects encountered. At the end of nine months, data was collected and results were evaluated using appropriate statistical methods.

\section{Statistical analysis}

Analysis was based on data obtained from patients who have completed nine months of study phase. Data generated from the study was tabulated with respect to all parameters at specific intervals. The results were expressed as mean $\pm \mathrm{SD}$ of each variable. Comparison between the two groups was done by unpaired T-test. All the statistical analysis was done using SPSS software. Significance was expressed as $\mathrm{p}$ value of $<0.05$ for each parameter.

\section{RESULTS}

Among the radiological parameters, ultrasonography (USG) of left and right ovaries was conducted. Table 1 shows that the percentage change in left ovarian volume at 9 months was not statistically significant ( $\mathrm{p}$ value $>0.05$ ) between the two groups.

Table 2 shows that the percentage decrease in right ovarian volume was not statistically significant ( $\mathrm{p}$ value $>0.05$ ) among the two groups.

Figure 1 shows that there was no statistically significant reduction in luteinizing hormone $(\mathrm{LH}) /$ follicle-stimulating hormone (FSH) between the 2 groups ( $\mathrm{p}$ value $>0.05$ ).

Figure 2 shows that the percentage change at 9 months from baseline in the two study groups show statistically significant fall in patients on myoinositol and dchiroinositolin group 2 ( $\mathrm{p}$ value $<0.001)$.

Figure 3 shows that the percentage change of serum free testosterone from baseline among the group 1 and 2 show statistically significant decrease in group 2 ( $\mathrm{p}$ value $<0.001)$.

Table 3 shows that the percentage change in estrogen levels was statistically non-significant ( $\mathrm{p}$ value $<0.05$ ) among the two groups.

Figure 4 shows that the percentage change at 9 months from baseline was not statistically significant between the two groups ( $\mathrm{p}$ value $<0.05$ ). Table 4 shows that the values were significant statistically in metformin group ( $\mathrm{p}$ value $<0.05)$.

Table 1: Left ovarian volume in study groups.

\begin{tabular}{|c|c|c|c|c|c|}
\hline \multirow{2}{*}{ Time } & \multicolumn{2}{|c|}{ Group 1 (in cm $^{3}$ ) } & \multicolumn{2}{|c|}{ Group 2 (in $\left.\mathrm{cm}^{3}\right)$} & \multirow{2}{*}{ P value } \\
\hline & Mean & SD & Mean & SD & \\
\hline Baseline & 11.61 & 2.34 & 11.46 & 1.62 & 0.801 \\
\hline At 3 months & 10.42 & 2.02 & 10.30 & 1.56 & 0.821 \\
\hline At 6 months & 8.76 & 1.55 & 8.88 & 1.01 & 0.731 \\
\hline At 9 months & 7.24 & 1.41 & 7.53 & 0.81 & 0.381 \\
\hline$\%$ change at 3 months & 9.99 & 3.53 & 10.01 & 6.50 & 0.990 \\
\hline$\%$ change at 6 months & 23.79 & 7.68 & 21.98 & 6.05 & 0.360 \\
\hline$\%$ change at 9 months & 36.59 & 10.25 & 33.48 & 8.63 & 0.251 \\
\hline
\end{tabular}

p $>0.05$; Not significant; ${ }^{*} p<0.05$; Significant; ${ }^{*} \mathrm{p}<0.001$; Highly significant. 
Table 2: Right ovarian volume in study groups.

\begin{tabular}{|llllll|}
\hline \multirow{2}{*}{ Time } & Group $1\left(\right.$ in $\left.\mathrm{cm}^{\mathbf{3}}\right)$ & \multicolumn{2}{l|}{ Group 2 $\left(\mathrm{in}^{\mathbf{3}}\right)$} & P value \\
\hline Baseline & Mean & SD & Mean & SD & 0.016 \\
\hline At 3 months & 11.12 & 2.34 & 12.58 & 1.72 & 0.040 \\
\hline At 6 months & 9.90 & 2.02 & 11.03 & 1.73 & 0.044 \\
\hline At 9 months & 8.40 & 1.48 & 9.21 & 1.31 & 0.135 \\
\hline \% change at 3 months & 6.92 & 1.40 & 7.40 & 0.73 & 0.124 \\
\hline \% change at 6 months & 10.71 & 4.35 & 12.52 & 3.81 & 0.112 \\
\hline \% change at 9 months & 23.54 & 8.24 & 26.63 & 4.82 & 0.152 \\
\hline
\end{tabular}

p $>0.05$; Not significant; $* \mathrm{p}<0.05$; Significant; $* * \mathrm{p}<0.001$; Highly significant.

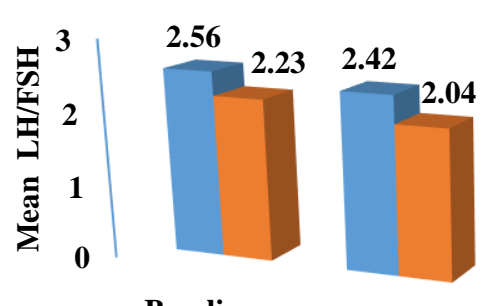

Baseline

At 3 months

TIME

At 6 months

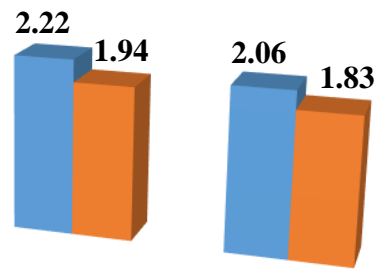

At 9 months

Figure 1: LH/FSH ratio in patients in study groups.

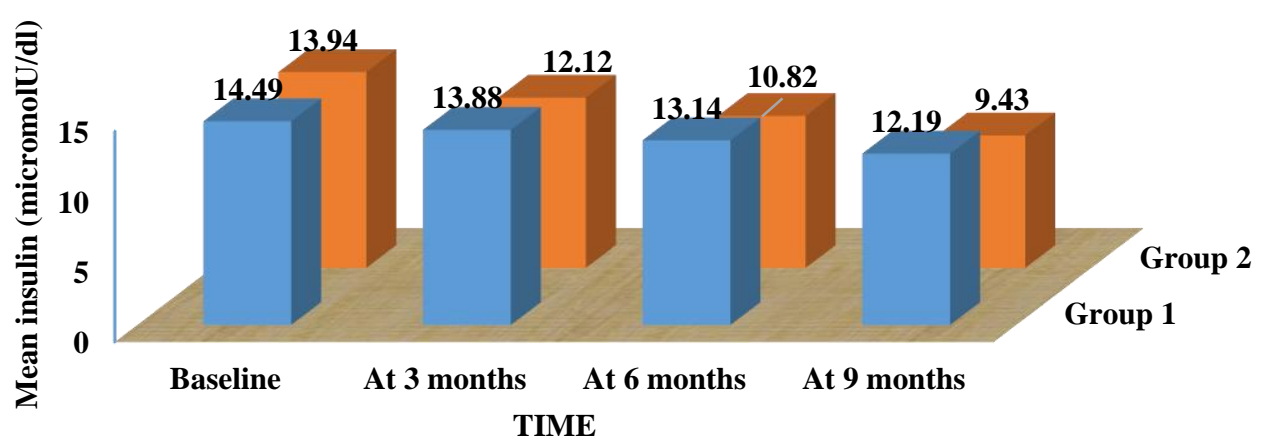

Figure 2: Serum insulin levels in micro IU/dl in patients in study groups.

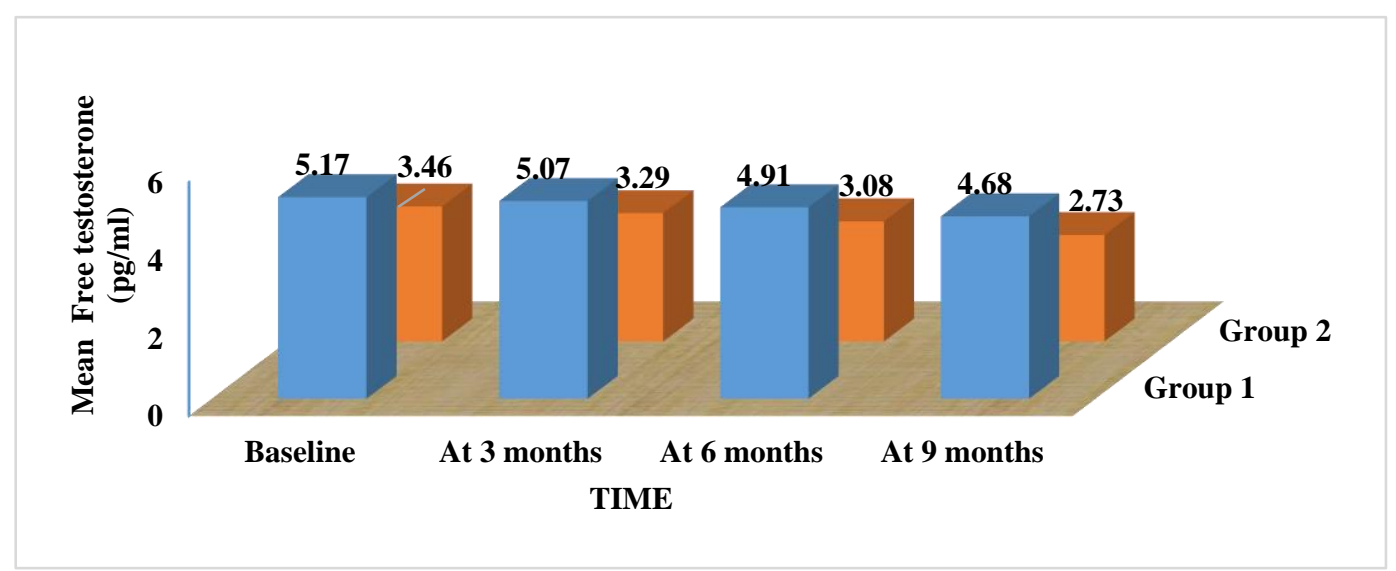

Figure 3: Serum free testosterone levels in $\mathrm{pg} / \mathrm{ml}$ in patients in study groups. 
Table 3: Serum estrogen levels in patients in study groups.

\begin{tabular}{|llllll|}
\hline \multirow{2}{*}{ Time } & Group $1(\mathrm{in} \mathrm{pg/ml})$ & \multicolumn{2}{l|}{ Group 2 $(\mathrm{in} \mathrm{pg/ml})$} & P value \\
\hline Baseline & Mean & SD & Mean & SD & 0.026 \\
\hline At 3 months & 61.35 & 18.96 & 50.68 & 13.50 & 0.023 \\
\hline At 6 months & 60.18 & 18.62 & 49.46 & 13.32 & 0.032 \\
\hline At 9 months & 59.08 & 18.54 & 49.15 & 12.76 & 0.030 \\
\hline \% change at 3 months & 57.96 & 18.19 & 48.07 & 12.50 & 0.096 \\
\hline \% change at 6 months & 1.89 & 1.17 & 2.54 & 1.52 & 0.121 \\
\hline \% change at 9 months & 3.85 & 1.69 & 2.74 & 3.09 & 0.406 \\
\hline
\end{tabular}

$\mathrm{p}>0.05$; Not significant; *p<0.05; Significant; ${ }^{* *} \mathrm{p}<0.001$; Highly significant.

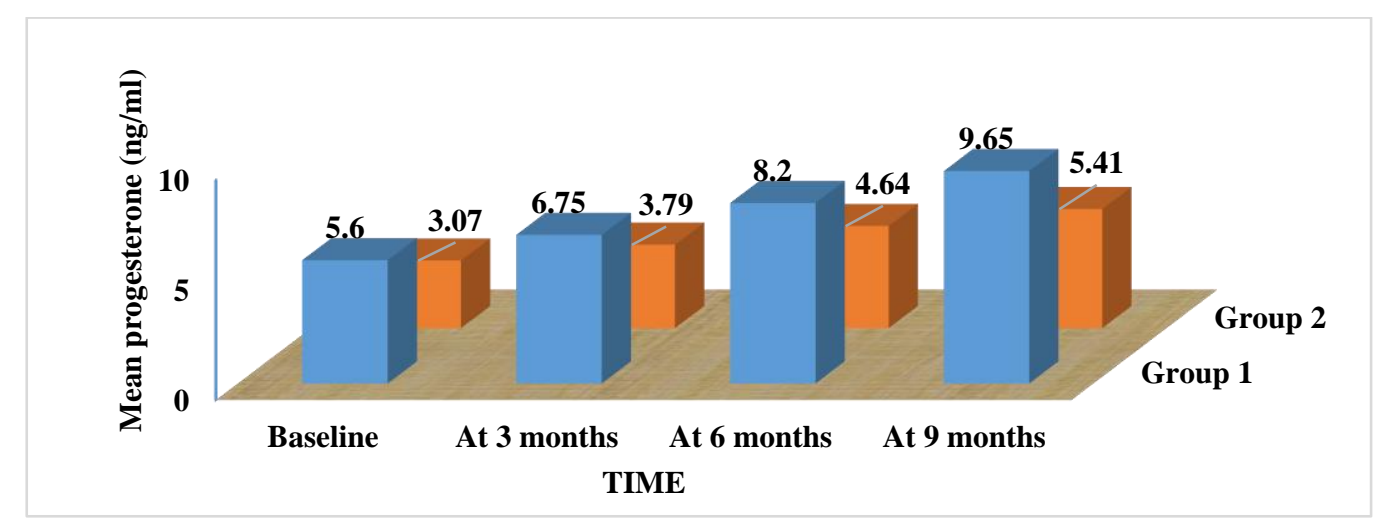

Figure 4: Serum progesterone levels in $\mathrm{ng} / \mathrm{ml}$ in patients in in study groups.

Table 4: Serum anti-mullerian hormone levels (AMH) in patients in in study groups.

\begin{tabular}{|llllll|}
\hline & \multicolumn{2}{l}{ Group $\mathbf{1}(\mathrm{in} \mathbf{n g} / \mathbf{m l})$} & \multicolumn{2}{l|}{ Group 2 $(\mathbf{i n}$ ng/ml) } & P value \\
\hline Time & Mean & SD & Mean & SD & 0.000 \\
\hline At 3 months & 9.47 & 2.15 & 15.75 & 3.30 & 0.000 \\
\hline At 6 months & 8.85 & 2.12 & 15.26 & 3.22 & 0.000 \\
\hline At 9 months & 8.11 & 2.14 & 14.46 & 3.22 & 0.000 \\
\hline \% change at 3 months & 7.39 & 2.06 & 13.65 & 3.15 & 0.001 \\
\hline \% change at 6 months & 6.68 & 4.40 & 3.12 & 1.86 & 0.000 \\
\hline \% change at 9 months & 14.85 & 7.07 & 8.38 & 2.73 & 0.000 \\
\hline
\end{tabular}

$\mathrm{p}>0.05$; Not significant; $* \mathrm{p}<0.05$; Significant; $* * \mathrm{p}<0.001$; Highly significant.

\section{DISCUSSION}

On ultrasonography, it was observed that the mean left ovarian volume at baseline in group 1 was $11.61 \pm 2.34$ and $11.46 \pm 1.62$ in group 2. This volume decreased to $7.24 \pm 1.41$ and $7.53 \pm 0.81$ after final follow up at 9 months in group 1 and 2 respectively. The percentage change at 9 months from baseline was $36.59 \pm 10.25$ in metformin groupand $33.48 \pm 8.63$ in myoinositol and d-chiroinositol group. The mean right ovarian volume at baseline was $11.12 \pm 2.34$ and $12.58 \pm 1.72$ which decreased to $6.92 \pm 1.40$ and $7.40 \pm 0.73$ after final follow up at 9 months in group 1 and 2 respectively. The percentage change at 9 months from baseline was 36.59 \pm 11.29 in Metformin group and $40.46 \pm 6.96$ in combination therapy group. The results were not statistically significant ( $\mathrm{p}$ value $>0.05$ ) between the two groups. Similar results have been reported by
Gharakhani et al and Zeyneloglu et al where decrease in ovarian volume was observed with metformin treatment. ${ }^{6,7}$ Our results are not in consonance with the study conducted by Ozay et al where myoinositol administration resulted in significant decrease in ovarian volume. $^{8}$

The mean LH/FSH on day 1 was $2.56 \pm 0.49$ and $2.23 \pm 0.53$ which decreased to $2.06 \pm 0.47$ and $1.83 \pm 0.37$ after final follow up at 9 months in group 1 and 2 respectively. The percentage change at 9 months from initial value was $20.18 \pm 5.87$ and $16.89 \pm 8.58$ amongst the 2 groups. There was no statistically significant reduction in LH/FSH between the 2 groups ( $\mathrm{p}$ value $>0.05$ ). Similar results were reported in the study conducted by Artini et.al. which showed decrease in the levels of LH/FSH ratio with myoinositol and d-chiroinositol whereas Nehra 
et.al. has observed more reduction in LH/FSH ratio with metformin group compared to myoinositol. ${ }^{9,10}$

The mean free testosterone at the start of the study was $5.17 \pm 1.69$ and $3.46 \pm 1.39$ which decreased to $4.68 \pm 1.57$ and $2.73 \pm 1.23$ at 9 months in group 1 and 2 respectively. The percentage change from baseline was $9.31 \pm 11.99$ and $22.46 \pm 6.47$ in group 1 and 2 showing statistically significant decrease in group 2 ( $\mathrm{p}$ value $<0.05$ ). The results were consistent with the study conducted by Troisi et al, Ozay et al and Regidor et al presenting reduction in testosterone levels with the administration of myoinositol and d-chiroinositol. ${ }^{8,11,12}$ Tiwari et al also observed similar trends in testosterone levels at 6 months in metformin group. ${ }^{13}$

The mean random blood glucose at baseline was $108.84 \pm$ 11.08 and $87.07 \pm 4.48$ which decreased to $100.64 \pm 7.73$ and $82.57 \pm 2.26$ after final follow up at 9 months in group 1 and 2 respectively. The percentage change at 9 months from baseline was $7.14 \pm 5.90$ and $5.00 \pm 4.01$ which was not statistically significant among the two groups ( $\mathrm{p}$ value $>0.05$ ). Similar variation in the mean random blood glucose was observed with metformin in a study conducted by Pradas et al. ${ }^{14}$ In contrast to our study, Shokrpour et al in his study reported significant reduction in blood glucose levels with myoinositol supplementation. ${ }^{15}$

The mean insulin on day 1 was $14.49 \pm 5.48$ and $13.94 \pm 3.95$ in group 1 and 2 respectively. The levels showed marked decrease in values measured as $12.19 \pm 4.85$ and $9.43 \pm 4.36$ after final follow up at 9 months. The percentage change at 9 months from baseline was $16.26 \pm 7.99$ and $34.24 \pm 15.02$ in the two study groups showing statistically significant fall in patients on myoinositol and d-chiroinositol ( $p$ value $<0.05$ ). Our results are in consistence with the study conducted by Fruzzetti et al, where both metformin and myoinositol group presented with decrease in insulin levels after treatment. ${ }^{16}$ Similar results have been reported with the use of metformin and myoinositol on insulin levels by Pradas et al and Artini et al, respectively. ${ }^{10,14}$ The results of the study conducted by Shokrpour et al are in consonance with our results showing significant reduction in serum insulin levels with myoinositol supplementation. ${ }^{15}$

The mean AMH on the day of enrollment was 9.47 \pm 2.15 and $15.75 \pm 3.30$ in group 1 and 2 which decreased to $7.39 \pm 2.06$ and $13.65 \pm 3.15$ after final follow up at 9 months. The percentage change at 9 months from baseline was $22.41 \pm 7.78$ and $13.67 \pm 3.76$ in group 1 and 2 respectively. The values were significant statistically in metformin group ( $\mathrm{p}$ value $<0.05$ ). These results are in consonance with studies conducted by Madsen et al where AMH levels significantly decreased during metformin treatment. ${ }^{17}$ Another study by Tagliaferri et al showed significant decrease in AMH levels with metformin treatment compared to myoinositol. ${ }^{18}$
The mean estrogen at the beginning of the study was $61.35 \pm 18.96$ and $50.68 \pm 13.50$ and these decreased to $57.96 \pm 18.19$ and $48.07 \pm 12.50$ at 9 months in group 1 and 2 respectively. The percentage change at the end of the study from baseline levels was $5.62 \pm 2.30$ and $4.84 \pm 4.07$ in the two groups. The change was statistically nonsignificant ( $p$ value $<0.05)$ among the two groups. The mean progesterone rose to $9.65 \pm 3.90$ and $5.41 \pm 1.38$ in the two groups from the baseline values on day $1(5.60 \pm 1.76$ and 3.07 \pm 1.54 ). The percentage change at 9 months from baseline was $73.83 \pm 45.71$ and $98.89 \pm 62.27$ which was not statistically significant between the two groups ( $p$ value $<0.05)$. Similar results were seen in a study conducted by Ozay et al where myoinositol led to reduction in estrogen levels and increase in progesterone levels. ${ }^{8}$ Even Regidor et al demonstrated the increase in progesterone levels with myoinositol supplementation. ${ }^{12}$

Treatment in both the groups was found to be safe. Most patients receiving metformin complained of dyspepsia and few complained of nausea, loss of appetite and diarrhea during the treatment. In contrast, no gastrointestinal side effects were reported in the myoinositol group and d-chiroinositol group, except for one who reported of diarrhea, confirming the high tolerability of myoinositol plus d-chiroinositol combination.

\section{CONCLUSION}

Metformin and myoinositol plus d-chiroinositol combination resulted in improvement of both clinical and hormonal disturbances in PCOS patients. Myoinositol and d-chiroinositol supplementation confirmed higher tolerability and also resulted in statistically significant reduction in free testosterone and insulin levels than metformin. Since the present study was carried out for a short duration and on a small number of patients, more studies are required to establish the effects of myoinositol and d-chiroinositol combination therapy for effective treatment of PCOS.

\section{Funding: No funding sources Conflict of interest: None declared \\ Ethical approval: The study was approved by the Institutional Ethics Committee}

\section{REFERENCES}

1. Bharathi RV, Swetha S, Neerajaa J, Madhavica JV, Janani DM, Rekha SN, et al. An epidemiological survey: Effect of predisposing factors for PCOS in Indian urban and rural population. Middle East Fertility Society Journal. 2017;22(4):313-6.

2. Grundy SM. Obesity, metabolic syndrome, and coronary atherosclerosis. Circulation. 2002;105(23):2696-8.

3. Sun T, Heimark DB, Nguygen T, Nadler JL, Larner J. Both myo-inositol to chiro-inositol epimerase activities and chiro-inositol to myo-inositol ratios are 
decreased in tissues of GK type 2 diabetic rats compared to Wistar controls. Biochem Biophys Res Commun. 2002;293(3):1092-8.

4. Nestler JE, Unfer V. Reflections on inositol(s) for PCOS therapy: steps toward success. Gynecol Endocrinol Off $\mathrm{J}$ Int Soc Gynecol Endocrinol. 2015;31(7):501-5.

5. Fauser BCJM, Tarlatzis BC, Rebar RW, Legro RS, Balen AH, Lobo R, et al. Consensus on women's health aspects of polycystic ovary syndrome (PCOS): the Amsterdam ESHRE/ASRM-Sponsored 3rd PCOS Consensus Workshop Group. Fertil Steril. 2012;97(1):28-38.e25.

6. Gharakhani M, Neghab N, Farimani M. Is Reducing Ovarian Volume in Polycystic Ovarian Syndrome Patients after Administration of Metformin Associated with Improving Cardiovascular Risk Factors? Int J Fertil Steril. 2011;5(2):90-5.

7. Zeyneloglu HB, Haydardedeoglu B, Bagis T, Kilicdag E. Metformin decreases ovarian volume in lean patients with polycystic ovary syndrome (PCOS). Fertil Steril. 2004;82:S301.

8. Ozay AC, Emekci Ozay O, Okyay RE, Cagliyan E, Kume T, Gulekli B. Different effects of myoinositol plus folic acid versus combined oral treatment on androgen levels in PCOS women. International $\mathbf{J}$ Endocrinol. 2016; 2016.

9. Nehra J, Kaushal J, Singhal SR, Ghalaut VS. A comparative study of myo inositol versus metformin on biochemical profile in polycystic ovarian syndrome in women. Int $\mathbf{J}$ Pharmaceut Sci Res. 2017;8(4):1664.

10. Artini PG, Di Berardino OM, Papini F, Genazzani AD, Simi G, Ruggiero $M$, et al. Endocrine and clinical effects of myo-inositol administration in polycystic ovary syndrome. A randomized study. Gynecol Endocrinol Off J Int Soc Gynecol Endocrinol. 2013;29(4):375-9.

11. Troisi J, Cinque C, Giugliano L, Symes S, Richards $\mathrm{S}$, Adair D, et al. Metabolomic change due to combined treatment with myo-inositol, D-chiroinositol and glucomannan in polycystic ovarian syndrome patients: a pilot study. J Ovarian Res. 2019; 12(1):25.
12. Regidor PA, Schindler AE. Myoinositol as a safe and alternative approach in the treatment of infertile PCOS women: a German observational study. Int $\mathbf{J}$ Endocrinol. 2016.

13. Tiwari N, Pasrija S, Jain S. Randomised controlled trial to study the efficacy of exercise with and without metformin on women with polycystic ovary syndrome. Eur J Obstet Gynecol Reprod Biol. 2019;234:149-54.

14. Pradas I, Rovira-Llopis S, Naudí A, Bañuls C, Rocha M, Hernandez-Mijares A, et al. Metformin induces lipid changes on sphingolipid species and oxidized lipids in polycystic ovary syndrome women. Sci Rep. 2019;9(1):16033.

15. Shokrpour M, Foroozanfard F, Ebrahimi FA, Vahedpoor Z, Aghadavod E, Ghaderi A, et al. Comparison of myo-inositol and metformin on glycemic control, lipid profiles, and gene expression related to insulin and lipid metabolism in women with polycystic ovary syndrome: a randomized controlled clinical trial. Gynecol Endocrinol. 2019;35(5):406-11.

16. Fruzzetti F, Perini D, Russo M, Bucci F, Gadducci A. Comparison of two insulin sensitizers, metformin and myo-inositol, in women with polycystic ovary syndrome (PCOS). Gynecol Endocrinol Off J Int Soc Gynecol Endocrinol. 2017;33(1):39-42.

17. Madsen HN, Lauszus FF, Trolle B, Ingerslev HJ, Tørring N. Impact of metformin on anti-Müllerian hormone in women with polycystic ovary syndrome: a secondary analysis of a randomized controlled trial. Acta Obstet Gynecol Scand. 2015;94(5):547-51.

18. Tagliaferri V, Romualdi D, Immediata V, Cicco SD, Florio CD, Lanzone A, et al. Metformin vs myoinositol: which is better in obese polycystic ovary syndrome patients? A randomized controlled crossover study. Clin Endocrinol (Oxf). 2017;86(5):725-30.

Cite this article as: Jaura S, Kaur I, Singh J, Madan A. A prospective study of metformin versus myoinositol plus d-chiroinositol combination therapy in polycystic ovarian syndrome. Int J Basic Clin Pharmacol 2020;9:276-81. 